\title{
Civilisations
}

Revue internationale d'anthropologie et de sciences

humaines

52-1 | 2004

Transmission des savoirs et interactions culturelles

\section{Une frontière, entre géographie et histoire...}

Archéologie des échanges et influences, dans les Andes équatoriales, à la période Formative

Jean Guffroy

\section{OpenEdition}

\section{Journals}

Édition électronique

URL : http://journals.openedition.org/civilisations/742

DOI : $10.4000 /$ civilisations.742

ISSN : 2032-0442

\section{Éditeur}

Institut de sociologie de l'Université Libre de Bruxelles

\section{Édition imprimée}

Date de publication : 1 mai 2004

Pagination : 61-80

ISSN : 0009-8140

\section{Référence électronique}

Jean Guffroy, «Une frontière, entre géographie et histoire... », Civilisations [En ligne], 52-1 | 2004, mis en ligne le 28 janvier 2009, consulté le 02 juin 2020. URL : http://journals.openedition.org/civilisations/ 742 ; DOI : https://doi.org/10.4000/civilisations.742 


\title{
Une frontière, entre géographie et histoire...
}

\author{
Archéologie des échanges et influences, \\ dans les Andes équatoriales, à la période Formative
}

\section{JEAN GUFFROY}

La zone de basses Andes comprise entre les quatrième et sixième degrés de latitude sud présente un ensemble de caractères géographiques et de développements historiques singuliers, qui en font un exemple pertinent pour l'analyse du concept de frontière, et l'étude, plus générale, des relations entre systèmes environnementaux, aires culturelles et structures sociopolitiques.

Cette région (Fig. 1), située entre les villes actuelles de Cuenca (Equateur) et de Cajamarca (Pérou), correspond à un point d'inflexion majeur de la Cordillère, ayant pour principales conséquences : un net abaissement des sommets, un élargissement de la chaîne andine et une fragmentation des vallées et des paysages. Elle occupe également une position intermédiaire entre les climats équatoriaux, du nord, et ceux des Andes et de la côte péruviennes, avec une diversification très marquée des écosystèmes (côte à mangrove, au nord/côte désertique, au sud ; Andes de Paramo/Andes de Puna, Ceja de montaña très humide à sèche).

Ces caractères singuliers, renforcés par des considérations géopolitiques modernes, ont fondé la réputation de zone relativement inhospitalière, inapte aux développements des grandes civilisations andines. Cette frontière anthropogéographique aurait provoqué un développement culturel séparé des grandes aires culturelles septentrionales et méridionales.

Les recherches archéologiques réalisées durant les vingt dernières années dans cette aire et les régions voisines, ont permis de reconstituer un panorama des occupations précolombiennes qui infirme sur plusieurs points cette vision déterministe, et permet une meilleure compréhension des singularités géographiques et historiques. Ces travaux ont, en particulier, mis en évidence l'existence d'un important essor culturel durant la période dite Formative (localement : 2000-300 BC), durant laquelle la position intermédiaire de la région paraît être un facteur clé du développement. On observe également des évolutions postérieures diverses, marquées à plusieurs moments par des mouvements, apparemment importants, de population. Cette reconstitution témoigne, aussi, de la complexité de cette problématique, qui est marquée par le poids des héritages, des situations géopolitiques et des interprétations savantes, et dont les paramètres sont distribués entre dynamiques et stratégies de développement, structures territoriales, limites linguistiques et identités culturelles. 


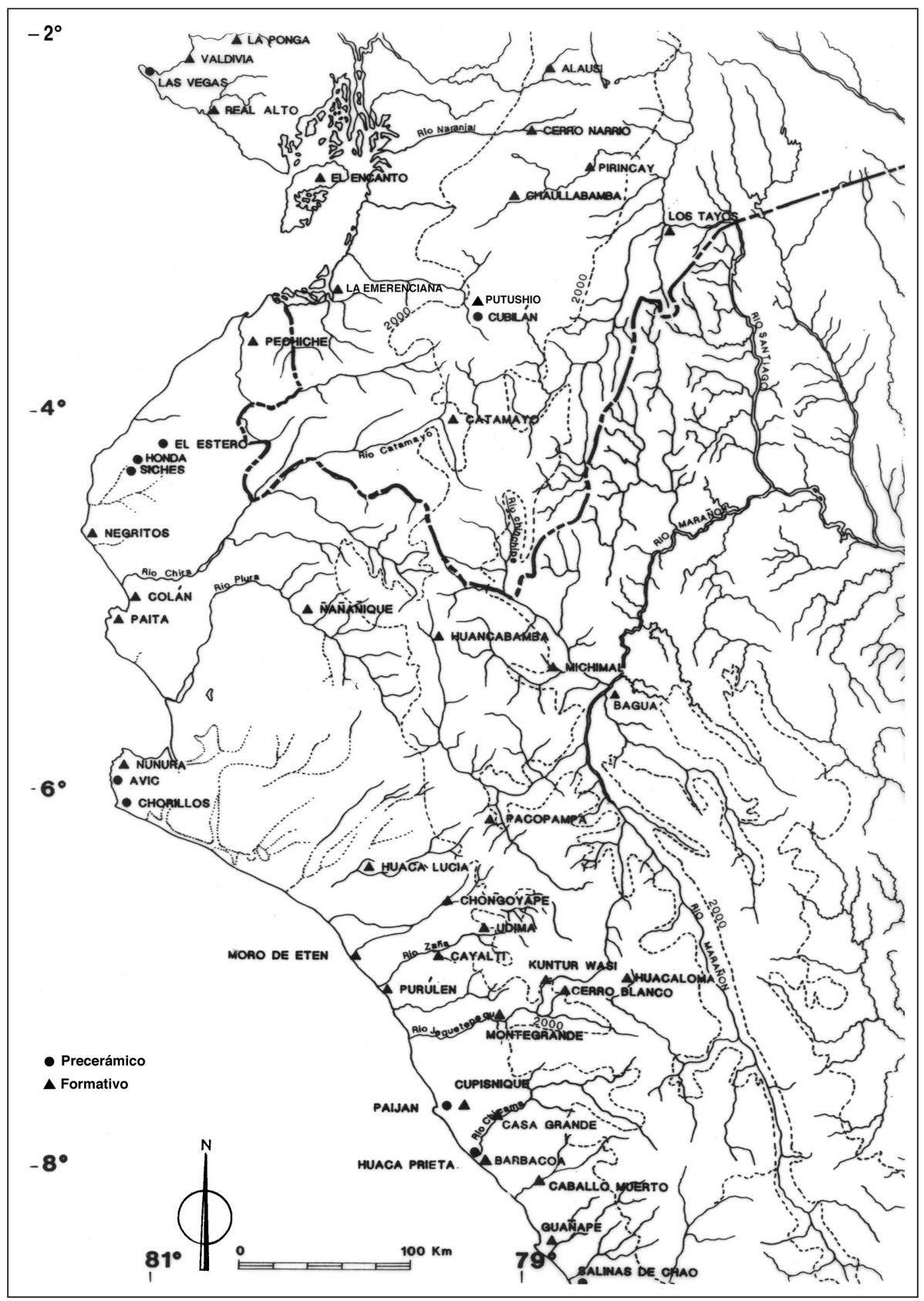

Fig. 1

Carte de la région et emplacement des principaux établissements cités dans le texte. 


\section{LES TERMES DE LA PROBLÉMATIQUE}

\section{Frontière anthropogéographique et culturelle ou espace de communication?}

L'hypothèse d'une frontière anthropogéographique a été soutenue, ces dernières décennies, par différents auteurs, avec des points de vue divers et parfois contradictoires. Il est malheureusement impossible de présenter ici un exposé détaillé des différentes hypothèses, que nous nous contenterons donc de résumer. Fondamentalement, c'est l'importance des contraintes environnementales et de leur permanence qui est ici en question.

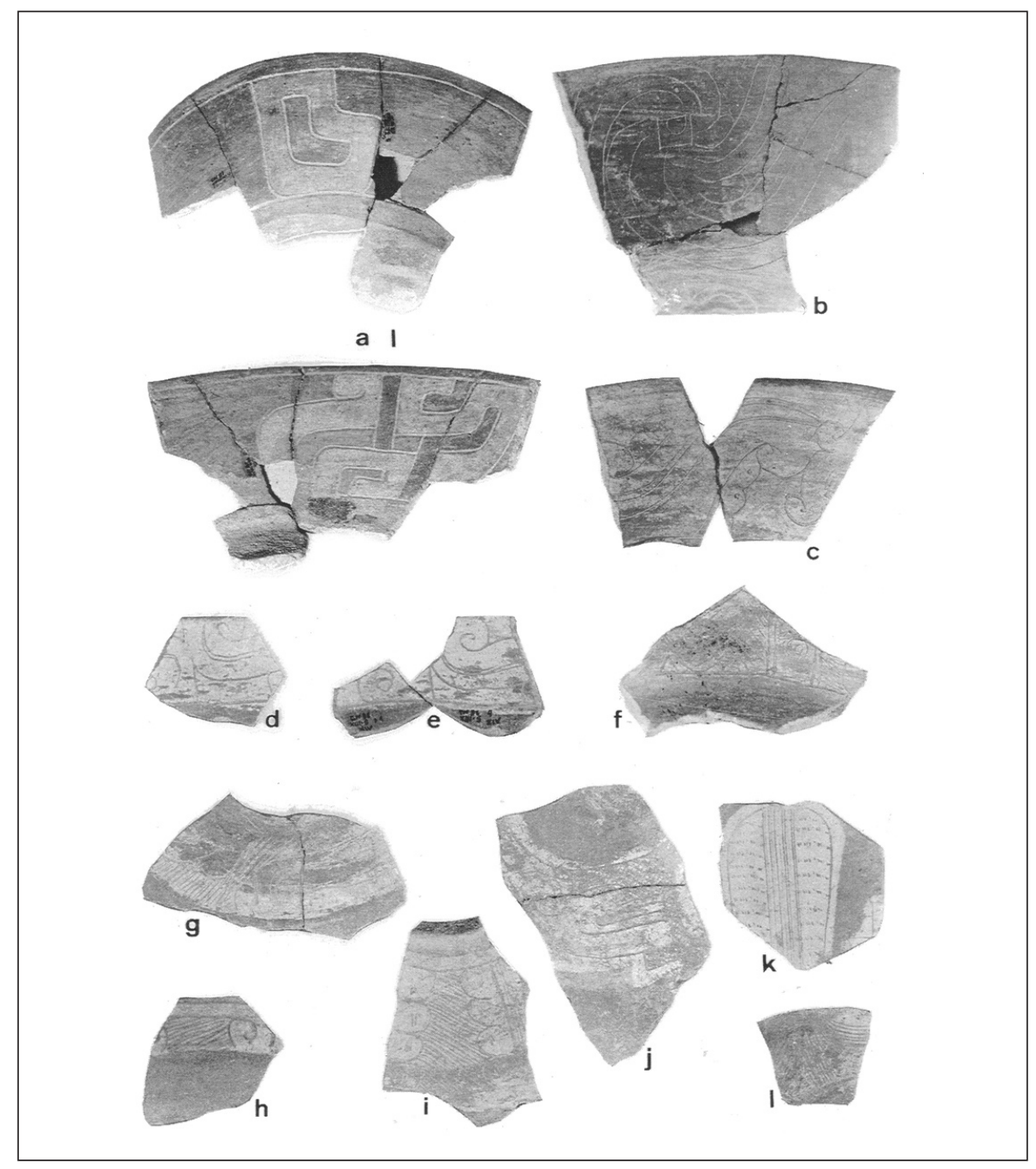

Fig. 2

Cerro Ñañanique : Matériel céramique de style A (phase Panecillo ; VII-Vè siècles BC). 
Dans un article paru en 1984, R. Burger, se fondant sur une thèse ancienne de W. Bennet (1948), a défini notre zone d'étude comme une frontière anthropogéographique, « ayant projeté les populations du Pérou et de l'Equateur actuel dans deux trajectoires de développement socioculturel et politique divergentes » (Burger 1984 : 53). Pour cet auteur : "Le désert de Sechura et les forêts insalubres de Catamayo constituent une zone naturelle de séparation, qui servit de base à une frontière de nature politique et anthropogéographique » (ibid. : 33). Selon lui : « ces aires sont incapables de supporter une population dense, équivalente à celle des régions de civilisation Chavín. La gradation de climat et des ressources entre les limites sud et nord des Andes de paramo est coupée par une région dont l'environnement est non attractif pour l'habitat humain. » (ibid. : 53). Les racines de cette divergence correspondraient à l'émergence de modes de production différents durant le second millénaire $\mathrm{BC}$. R. Burger a reconnu, plus récemment (1995 : 100-101), l'existence de liens et de relations unissant le Haut Piura à l'aire Cupisnique, ainsi que de nombreux contacts « à travers la très perméable zone frontière » (ibid. : 102), tout en maintenant son hypothèse initiale.

A.M. Hocquenghem (1991), reprenant en partie les thèses de Burger, considère la région d'étude comme extérieure à l'aire culturelle centre-andine, caractérisée par un système de production, une organisation sociale et un ordre idéologique singuliers. Cette frontière, qui résulterait de conditions naturelles défavorables (ibid. : 314), aurait connu, dans le courant des deux derniers millénaires, un certain déplacement résultant du développement des systèmes d'irrigation permettant une augmentation des terres cultivables. Plus récemment, dans un article écrit avec d'autres chercheurs travaillant au Pérou et en Equateur, le même auteur (Hocquenghem et al. 1993 : 449) révise quelque peu son analyse en décrivant le territoire d'étude comme une région de passage entre deux grandes aires culturelles, ayant maintenu son autonomie par rapport aux peuples voisins.

M. Tellenbach (1995), qui a également tenté de reconstituer les relations entre le Pérou et l'Equateur durant la période Formative, en se basant sur la distribution de quelques formes céramiques et de traits iconographiques singuliers, défend un point de vue sensiblement différent. Pour cet auteur (ibid. : 281), ces données stylistiques indiqueraient qu'il existe un moment historique durant lequel le sud de l'Equateur fut intensément associé au monde culturel «Chavín-Ofrendas » (IX-VIII" siècles BC). Ces liens se seraient relâchés postérieurement, au Formatif final, lors de la non-intégration du sud équatorien au monde idéologique «Chavín-Paracas » (phase Janabarriu, V-III ${ }^{\mathrm{e}}$ siècles $\mathrm{BC}$ ), qui modèlera de son empreinte l'intégration centre andine postérieure. Il admet toutefois la diffusion de certains traits Janabarriu jusqu'à Loja (ibid. : 288).

Nous avons, dans plusieurs travaux antérieurs (Guffroy 1995 ; Guffroy et al. 1987, 1994), discuté l'aspect par trop schématique d'une partie de ces reconstitutions, faussées à notre avis par différents facteurs :

a. un état des connaissances encore parcellaire, et inégalement abouti suivant les régions ;

b. une application trop stricte du déterminisme écologique et géographique, basée sur une vision tronquée et dépréciative des ressources et potentialités agricoles réelles de nombreux secteurs ;

c. une absence de prise en compte des données archéologiques récentes ; 


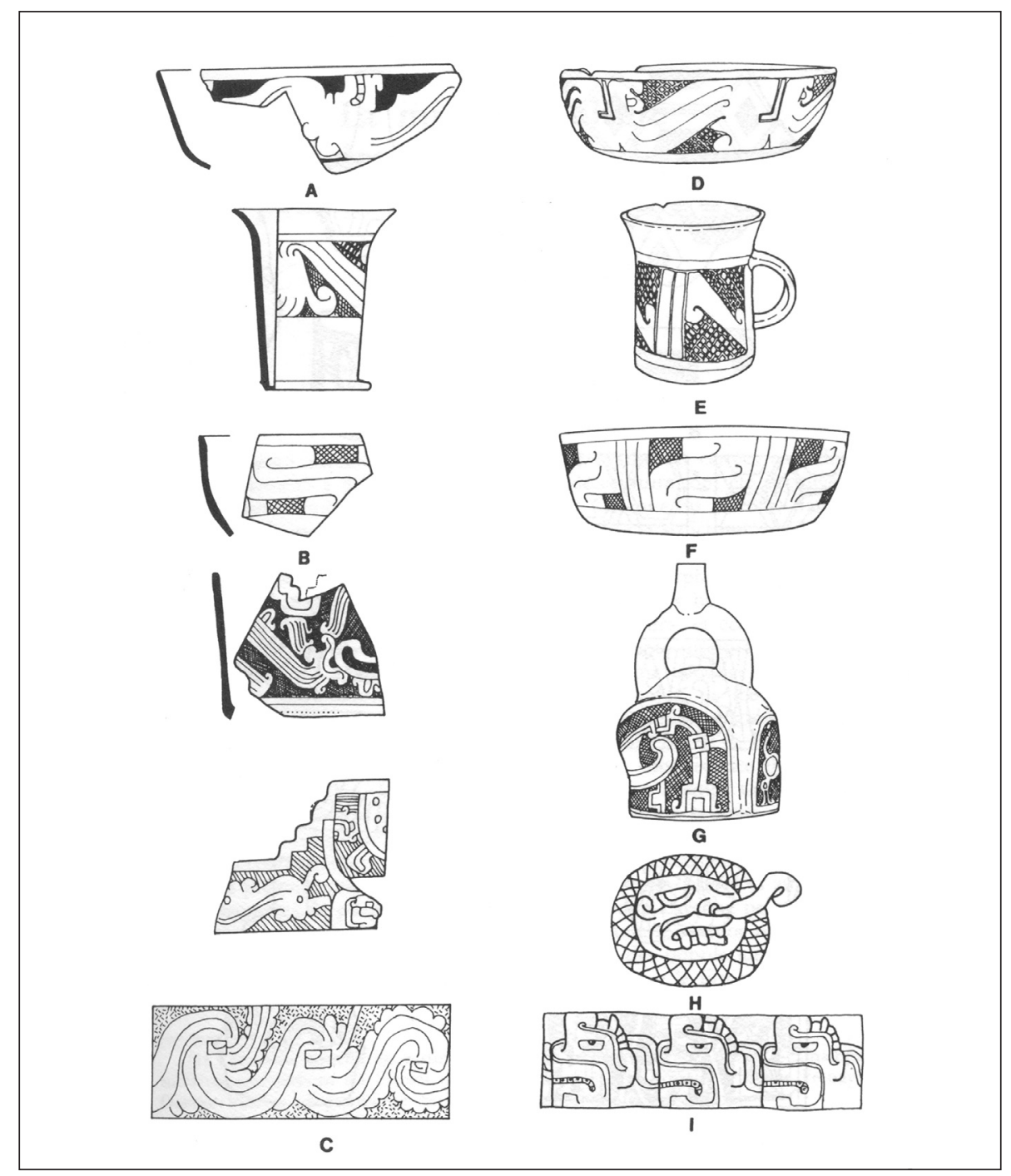

Fig. 3

Comparaison de l'iconographie des styles Local $B(A, B)$ et Local $A(C)$ avec des pièces provenant des vallées de Zaña y Jequetepeque (D, G-I) et d'une représentation murale du temple de Garagay (H).

d. une vision simplificatrice de la nature et du fonctionnement possible d'une zone frontalière, trop fréquemment réduite à une simple ligne délimitant, sur une carte, deux portions de territoire.

En ce qui concerne plus précisément la période Formative, sur laquelle se sont focalisées les discussions, on peut tout particulièrement critiquer :

- une sous-évaluation des potentialités de la région, tant en terme de production que d'échange des ressources.

- une généralisation souvent abusive des situations les mieux connues et une faible 
prise en compte des évolutions socio-culturelles importantes intervenues entre $2000 \mathrm{BC}$ et $300 \mathrm{BC}$. On ne peut préjuger du développement d'une région sur la base du développement d'une seule phase ou d'une seule portion de vallée.

- une surévaluation de l'homogénéité culturelle des régions avoisinantes et, tout particulièrement, de l'intégration sociopolitique de l'aire idéologique Chavín (si l'on excepte la courte période du Formatif final (V-III' siècles BC)).

- une fixation sur les termes nord-sud des relations, alors que les contacts est-ouest sont également fondamentaux.

\section{QUELQUES DONNÉES PERTINENTES}

Nous présenterons, dans la suite de cet article, des données correspondant à différentes sphères d'activités sociales qui permettent de mieux caractériser la complexité des phénomènes intervenus durant les derniers millénaires de l'époque préhispanique. Notre but premier est de fournir des éléments de réflexion pour une analyse des modes de fonctionnement d'une zone réputée frontalière, au moment de l'apparition des premières grandes civilisations andines. Cette présentation, qui ne se veut pas exhaustive, insistera plus particulièrement sur les phénomènes qui témoignent de la complexité des relations existant entre les différents acteurs sociaux régionaux, le plus souvent encore mal comprises. Nous tenterons enfin de démontrer, au moyen de l'analyse des données recueillies lors des recherches menées de part et d'autre de la frontière séparant actuellement le Pérou et l'Equateur, entre 1979 et 1990, le caractère par trop schématique et idéologique de la notion de frontière culturelle telle qu'elle est fréquemment utilisée.

\section{Peuplements / Phases culturelles}

Les premiers agriculteurs sédentaires installés dans la vallée de Catamayo (Province de Loja - Equateur) (Fig. 1), dans les premiers siècles du second millénaire $\mathrm{BC}$, possèdent un matériel céramique qui diffère notablement des styles contemporains de la côte et des Andes équatoriennes et péruviennes (Guffroy et al. 1987). Cette absence de relation témoigne sans doute de phénomènes de colonisation à partir de secteurs encore inconnus archéologiquement, tels que le piémont ou la forêt amazonienne. Elle met également en évidence l'état très parcellaire de nos connaissances.

Une rupture intervient vers les XIII-XII ${ }^{\mathrm{e}}$ siècles BC, avec l'apparition d'un nouveau matériel céramique, qui paraît refléter l'arrivée d'un nouveau groupe de population. Les formes associées semblent se rattacher au matériel utilitaire de la côte (culture Machalilla). De nouvelles évolutions interviennent vers le $\mathrm{IX}^{\mathrm{e}}$ siècle $\mathrm{BC}$, époque à laquelle apparaissent localement de fortes influences stylistiques provenant de la côte et surtout de la sierra équatorienne (cultures Chorrera et Chaullabamba/Cerro Narrio). Ces relations sont confirmées par la présence de pièces importées de ces régions septentrionales. L'axe privilégié de ces échanges et influences change d'orientation aux alentours du $V^{\mathrm{e}}$ siècle BC. Les formes, les techniques décoratives et l'iconographie, qui font alors leur apparition dans la vallée de Catamayo, sont directement influencées par les cultures méridionales contemporaines (traditions Pacopampa et Cupisnique). 


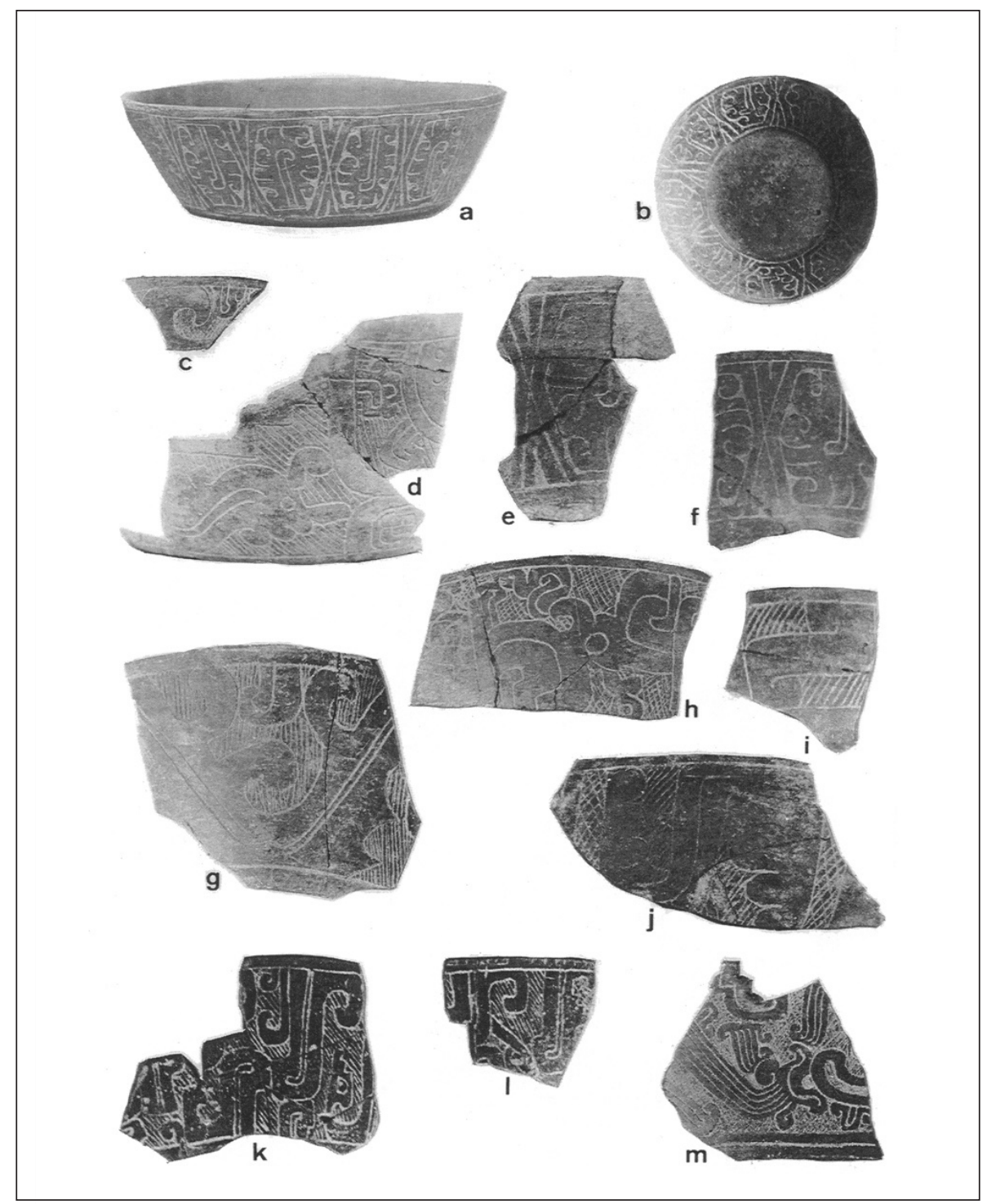

Fig. 4

Cerro Nañañique : Matériel céramique de style B (phase Panecillo).

Dans la vallée plus méridionale du rio Piura, à proximité de la ville actuelle de Chulucanas (département de Piura - Pérou) (Fig. 1), les plus anciens vestiges découverts à ce jour datent du XIII ou XII ${ }^{\mathrm{e}}$ siècle BC (Guffroy et al. 1995). L'occupation de ce secteur de piémont, situé en limite de désert, semble être lié à la position géographique du lieu, oasis naturelle située au carrefour de voies de communication. Elle paraît s'être accompagnée de l'émergence d'un pouvoir local, matérialisé par l'implantation d'un centre civico-cérémoniel, qui connaîtra un accroissement notable postérieurement au VII ${ }^{\mathrm{e}}$ siècle BC (Fig. 7). Dès le début de l'occupation, le matériel céramique 
de ce secteur se caractérise par la présence de deux styles bien différenciés (Fig. 2, 4), dont la coexistence pourrait éventuellement traduire un système de structuration sociale duale, ou un peuplement pluriculturel. Un des styles est utilisé dans la décoration des jarres et de bols usuels, alors que l'autre semble plutôt réservé à la fabrication de plats et de bols de libation, souvent finement décorés. Les importantes évolutions architecturales, qui interviennent aux alentours du $\mathrm{VII}^{\mathrm{e}}$ siècle $\mathrm{BC}$, s'accompagnent d'une évolution du matériel céramique marquée par un accroissement des relations stylistiques et iconographiques avec la côte et les Andes plus méridionales (Fig. 3). Une nouvelle rupture, qui voit l'abandon du centre cérémoniel, sans doute précédé de son incendie, intervient au $\mathrm{V}^{\mathrm{e}}$ siècle $\mathrm{BC}$. Les traditions céramiques antérieures sont, pour l'essentiel, délaissées et l'on note l'adoption de formes et de motifs décoratifs qui semblent témoigner d'un renforcement des influences méridionales.

Ces deux schémas d'évolution, sensiblement différents, confirment en premier lieu de l'existence de situations complexes, dans lesquelles interviennent des spécificités locales et des mouvements socio-idéologiques de plus grande ampleur, déterminant les systèmes de relation. Quelles que soient les incertitudes relatives à la nature et aux vecteurs des influences, ces données ne confortent en aucun cas la vision d'une zone isolée et sous-développée.

\section{Les formes et styles céramiques}

Chacun des secteurs de la région présente des assemblages céramiques sensiblement différents, dont la distribution répond à des facteurs jusqu'à maintenant mal expliqués. On peut en premier lieu reconnaître une nette opposition entre les assemblages où prédominent les jarres de moyenne et petite dimensions, et les ensembles céramiques où les formes de bols et de bouteilles sont plus abondantes (Deboer 1995). Ces différences reflètent très probablement des traditions, et pratiques culturelles, diversifiées; mais également des modes de développement singuliers. L'usage de bouteilles et de récipients de libation finement décorés paraît plus particulièrement associé aux contextes rituels et funéraires, et aux sociétés présentant une certaine stratification sociale. A partir du $\mathrm{VII}^{\mathrm{e}}$ siècle $\mathrm{BC}$, la diffusion, en petites quantités, de ces pièces plus ou moins somptuaires, semble généralisée dans toute l'aire d'étude, mais leur fabrication locale reste souvent limitée. Une autre des adoptions et diffusions notables correspond à l'apparition, dans la vallée de Catamayo, au Ve siècle $\mathrm{BC}$, d'une forme de récipient typique de la côte et Andes centrales péruviennes (jarre sans col, à lèvre épaissie), forme qui disparaît à la période suivante. Son adoption locale résulte, et accompagne, les influences méridionales qui touchent alors la région. S'agissant d'un récipient d'usage utilitaire, elle permet également de s'interroger sur la nature des sphères d'activité (idéologiques et religieuses, mais aussi économiques et alimentaires), impliquées dans ces interactions culturelles.

La distribution régionale des différents styles céramiques, durant le dernier millénaire $\mathrm{BC}$ (Fig. 5), répond à des logiques que nous sommes loin de comprendre, et qui prennent sans doute leurs racines dans les mouvements de population et les restructurations sociales qui ont affecté toute la région à partir de $1500 \mathrm{BC}$. Les relations unissant les styles céramiques à des aires culturelles et à des groupes de population homogènes, qui paraissent assez étroites antérieurement au $\mathrm{XIII}^{\mathrm{e}}$ siècle $\mathrm{BC}$, semblent 


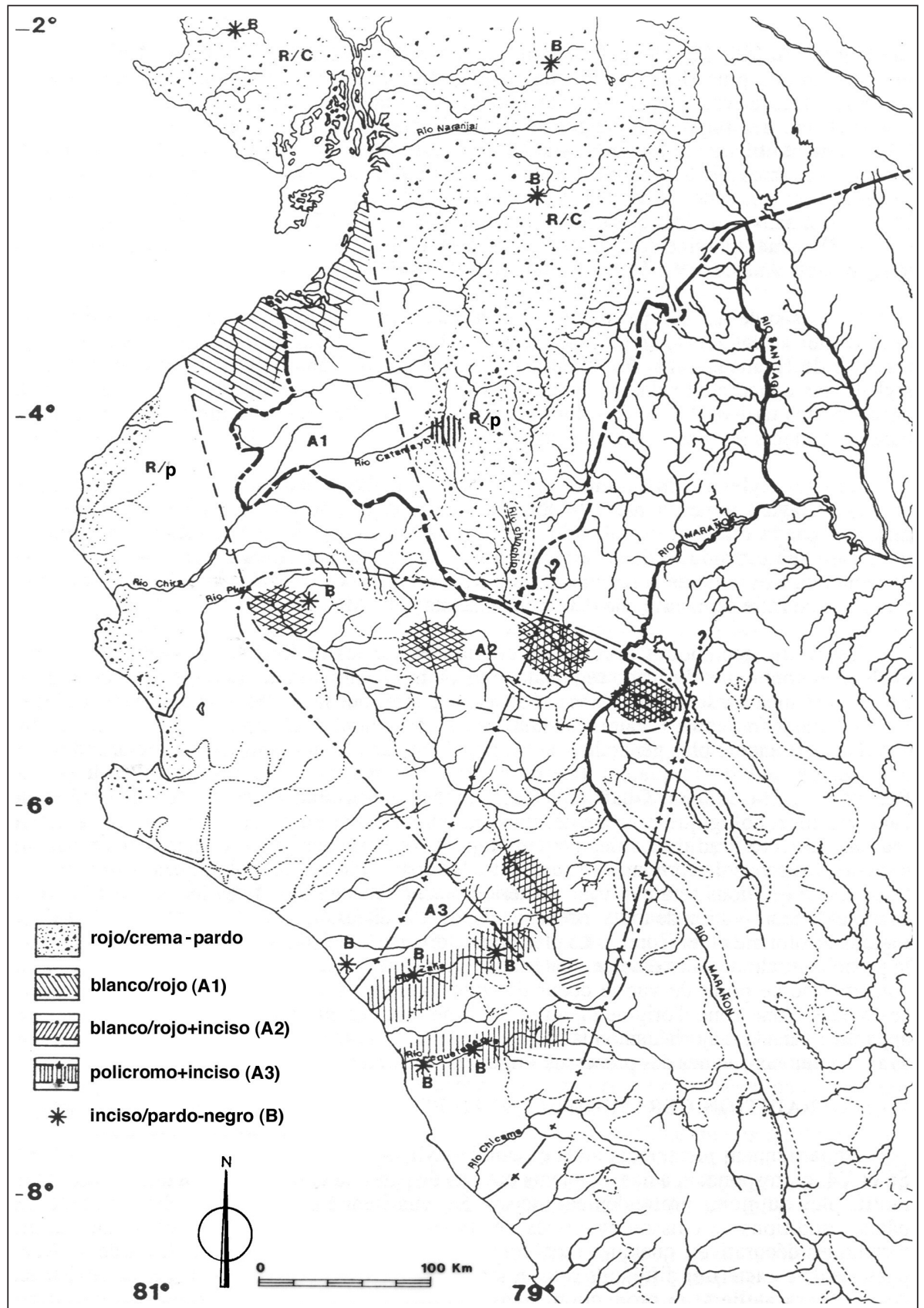

Fig. 5

Distribution des principaux styles céramiques régionaux entre le $I X^{e}$ et le $V^{e}$ siècle $B C$. 
alors connaître une importante évolution, sans doute sous l'effet conjugué de l'apparition de centres de pouvoir et d'un artisanat spécialisé. Ainsi, le style B (Fig. 4), qui constitue une composante importante du matériel céramique de Cerro Ñañañique, n'a été jusqu'à maintenant identifié dans aucun secteur environnant (Tumbes, Paita, Bagua Pacopampa), mais se rattache assez clairement à des styles équatoriens (Ayangue inciso, Alausi..), sans doute sur la base d'une filiation commune avec la tradition Valdivia finale. Il est également présent dans les vallées méridionales des rios Zaña et Jequetepeque (Alva, 1985), en tant que style mineur intégré à la production céramique Cupisnique. Dans le courant du $\mathrm{I}^{\text {er }}$ millénaire BC, ce style, clairement associé à la production d'objets de qualité dont les formes différent d'une région à l'autre (bols, coupes, bouteilles), connaît une vaste distribution, caractérisée par l'absence de continuité territoriale (Fig. 6). Toutefois, bien que des pièces isolées de ce style, porteuses d'une iconographie complexe, aient été retrouvées depuis Cuenca, au nord (Collier, Murra 1943 : pl. 10-4), jusqu'à Chavín de Huantar (Galerie des Offrandes), au sud (Lumbreras 1993 : fig. 654, 655), la vallée du Haut Piura est le seul secteur connu, à ce jour, où ce style constitue une composante majeure de la production céramique, produite sans interruption durant au moins sept siècles.

L'analyse de la distribution des récipients céramiques de style A (Fig. 2), associés à une plus grande diversité de formes, permet de mettre en évidence des différences de statut et de mode de diffusion entre les différents styles décoratifs, mais également des situations de compétition, reflétant sans doute des structurations sociales relativement complexes. La présence d'au moins deux styles céramiques (généralement un style peint et un style incisé) paraît plus ou moins généralisée à cette époque, tant dans l'aire stylistique Chaullabamba, que dans la zone orientale (Bagua) et méridionale (Pacopampa, Cupisnique). Dans le Haut Piura, cette coexistence semble résulter de la présence de deux groupes d'artisans, dont les productions sont bien différenciées tant du point de vue des pâtes que de la fabrication et de la cuisson (Guffroy et al. 1994 : 351-366). Cette production pourrait éventuellement témoigner d'un peuplement de caractère pluriethnique ou d'une division sociale par « moitiés ». Un matériel décoré suivant des modes décoratifs proches du style A est présent, vers l'est, à Huacabamba et Bagua et, vers le nord, à Pechiche (Fig. 5).

Une source d'erreur fréquente dans l'interprétation de la répartition des traits stylistiques tient à la primauté accordée à la recherche de traits communs tendant à fonder l'existence d'aires homogènes. Ainsi, s'il existe bien des relations stylistiques entre le matériel céramique produit, dans le courant du VIII ${ }^{\mathrm{e}}$ siècle $\mathrm{BC}$, à Catamayo, à Oña, situé à $60 \mathrm{~km}$ plus au nord, à Chaullabamba, à Pirincay ou encore à Cerro Narrio ; une analyse plus détaillée atteste de l'existence de nombreuses singularités, qui font des productions céramiques de chacun de ces secteurs des assemblages très sensiblement différents. Classer ces productions dans une même aire culturelle «Chaul-labamba » (Tellenbach 1995) paraît de ce fait quelque peu abusif, et par trop simplificateur. Une démonstration semblable pourrait être faite, au moins partiellement, pour les aires culturelles dites « Chorrera », « Cupisnique », « Pacopampa » ou «Chavín ». La nature du mode de production des différents récipients (ménager, artisanal, spécialisé...), primordial dans cette problématique, reste encore, pour l'essentiel, méconnue.

Ces différences locales attestent de singularités microrégionales, susceptibles de refléter des organisations sociales identitaires. Cette diversification volontaire semble 
perdre de l'importance au Formatif final, sans doute sous l'effet d'une relative intégration macrorégionale, et d'une réforme des systèmes de production céramique. Ces singularités témoignent également du caractère plus ou moins limité des interactions sociopolitiques antérieures, et d'une grande indépendance dans la nature, et modalités, des relations, échanges et influences. Si à Catamayo, comme à Chaullabamba, sont présents des vestiges céramiques témoignant de relations entre les populations du sud équatorien et celles du nord du Pérou, ces produits sont de nature diverse et attestent de contacts avec des régions sans doute bien différenciées du sud (aire Cupisnique pour Chaullabamba (Gomis 1999), aire de Pacopampa et versant oriental pour Catamayo (Guffroy 1995)).

L'étude de la répartition des différents motifs iconographiques, qui doit être menée en parallèle à celle des relations stylistiques, demande une discussion complexe, qui peut difficilement être présentée ici en quelques lignes. Cette analyse (Guffroy et al. 1994 : 313-339) confirme pour l'essentiel les données stylistiques, en montrant l'importance particulière accordée à l'expression d'une variation originale à partir de quelques thèmes communs. L'existence d'une figure centrale, à caractères monstrueux, est ainsi très largement répandue à cette époque.

\section{La circulation des produits céramiques}

L'étude de l'origine des fragments céramiques collectés sur les différents sites permet également de mettre en évidence certaines caractéristiques des systèmes d'échange. Une partie de ces pièces, provenant de régions plus ou moins éloignées, correspond à des récipients usuels contenant vraisemblablement des produits à échanger, ou accompagnant les voyageurs. Mais il s'agit également, et sans doute plus couramment durant le dernier millénaire $\mathrm{BC}$, de vases de qualité artistique, destinés à certaines pratiques, ainsi qu'à l'usage des élites locales. La différenciation de ces deux modes de transmission est facilitée par la nature différenciée des récipients (jarres et bols communs/bouteilles et bols finement décorés). Les récipients à iconographie complexe ont par ailleurs constitué (avec les textiles) un des supports privilégiés pour la diffusion des différentes influences idéologiques. Les publications archéologiques ont, malheureusement, trop longtemps traité sur un mode mineur les fragments céramiques dits « atypiques », présents en petit nombre dans les collections. Un autre écueil pour l'analyse de ces échanges tient aux difficultés à attribuer ces tessons à un lieu ou à un style d'origine bien déterminée. Dans la vallée de Catamayo, les fragments céramiques de ce type sont pratiquement totalement absents des niveaux des deux phases les plus anciennes, ce qui semble refléter un relatif isolement de la vallée, et/ou la rareté des échanges de ce type produit antérieurement au $\mathrm{I}^{\mathrm{er}}$ millénaire BC. Ces fragments de récipients importés sont au contraire particulièrement nombreux et divers dans les niveaux associés à la tradition postérieure Catamayo $\mathrm{C}$ (IX-VI ${ }^{\mathrm{e}}$ siècles BC). Prédominent les pièces de style Chaullabamba/Cerro Narrio (Guffroy 1985 : Fig. 14, i), provenant vraisemblablement de différents secteurs septentrionaux, mais il existe également d'autres fragments dont l'origine est, jusqu'à maintenant, inconnue. Durant la phase postérieure Catamayo D (V-IV siècles $\mathrm{BC})$, les récipients importés du nord semblent se raréfier, voir disparaître, au profit d'importation de matériel d'origine méridionale (ibid. : Fig. 18). La diversité de provenance paraît alors moindre, 
mais l'importance de l'échantillon actuellement collecté est trop réduite pour être vraiment significative.

L'analyse de ces mouvements de pièces céramiques est encore plus pertinente à Cerro Nañañique, tant en raison de l'importance de l'échantillon collecté (150000 tessons), que de la nature particulière du site. Ces fragments de récipients importés

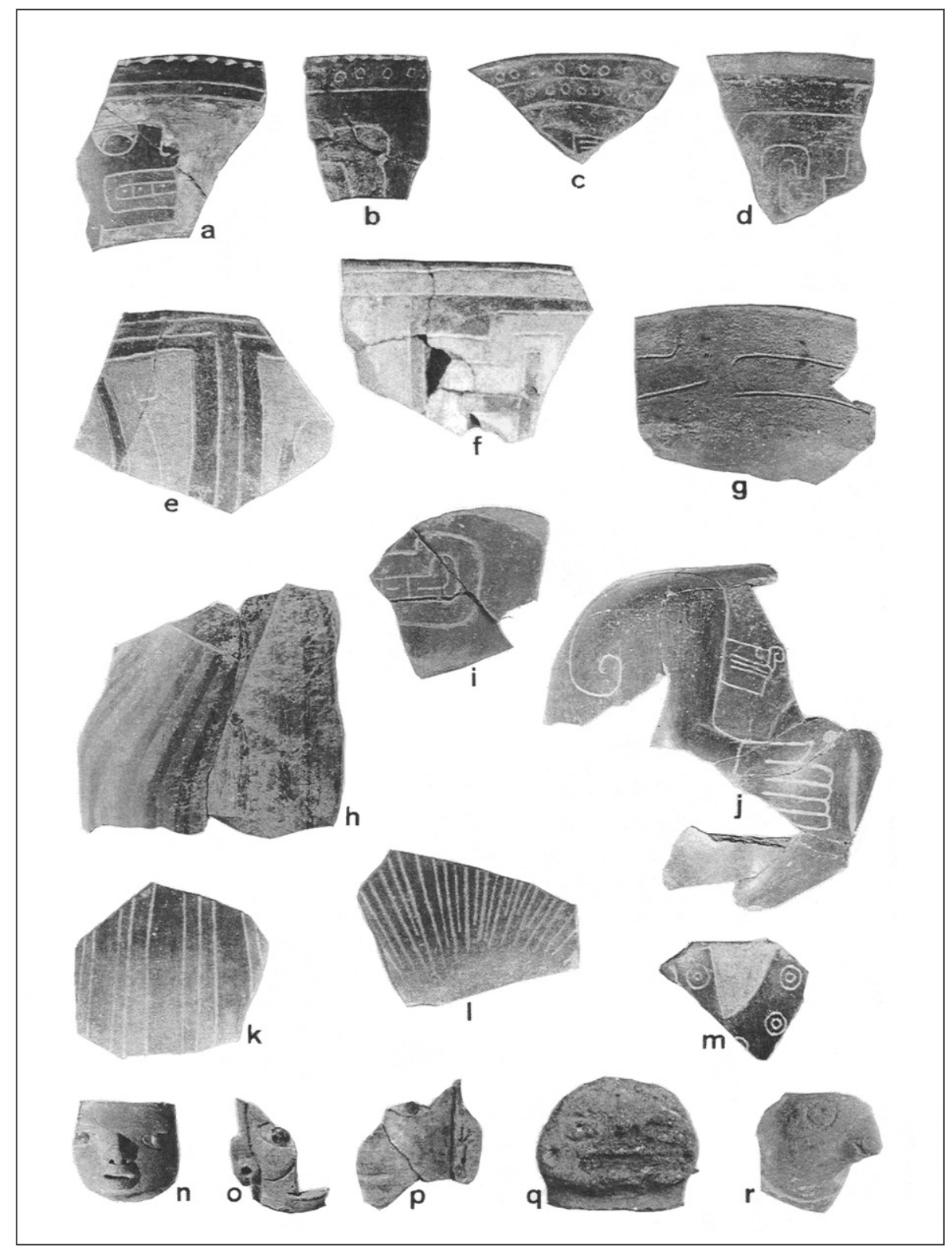

Fig. 6

Cerro Ñañañiqu : Vestiges de récipients importés, d'origines diverses.

Provenances vraisemblables : $a-d, n-p$ : vallées des rios Zaña et Reque ; e-f: Bagua, Pacopampa; $g$ : vallée du Jequetepeque ; $h-m$ : aire Cupisnique. 
se répartissent en différentes catégories. On peut, en premier lieu, reconnaître la présence de nombreuses variantes des deux styles de récipients locaux (A et B), produites dans des secteurs plus ou moins distants, avec des caractéristiques quelque peu singulières. Ces tessons prouvent l'existence de plusieurs groupes d'artisans, sans doute dispersés sur un assez vaste territoire, fabriquant et décorant des récipients suivant des canons stylistiques proches de ceux du Haut Piura. Un second groupe important de matériel correspond aux vases de style Paita, dont l'analyse de composition des pâtes (Guffroy et al. 1994 : 352-363) semble confirmer qu'ils ont été produits sur les sites côtiers, distants d'une centaine de kilomètres. L'utilisation d'un de ces récipients comme offrande paraît témoigner, au-delà de la simple fréquentation régulière du lieu, de l'intégration de ces populations de pêcheurs aux pratiques civiques et rituelles menées à bien sur le site.

On a reconnu également la présence de neuf styles régulièrement importés et de quatorze autres styles « rares », représentés par moins de cinq fragments. L'ensemble de ce matériel provient de plusieurs dizaines de zones d'origine réparties dans l'ensemble des régions voisines (Fig. 6). Les secteurs de provenance les mieux caractérisés correspondent aux différentes vallées méridionales : Reque, Zaña, Jequetepeque, ainsi qu'aux secteurs plus orientaux tels que Pacopampa et Bagua. Mais il existe également des pièces caractéristiques des traditions septentrionales (Pechiche, Chorrera, Chaullabamba, Catamayo C). Sur la base de ces données, on peut clairement affirmer la fréquentation du site par des groupes d'origines diverses, ainsi que les relations privilégiées existant avec les populations de la côte nord péruvienne, d'où ont été principalement importés des figurines, des bols et des bouteilles finement décorées.

\section{Les échanges de produits non céramiques}

Les échanges à courte et moyenne distances, permettant la complémentarité de l'exploitation de différents écosystèmes, sont sans doute inscrits depuis très longtemps dans la préhistoire sud-américaine. Ces trafics, qui ont probablement concerné, en premier lieu, des ressources alimentaires (poissons et coquillages séchés, graines, fruits et légumes cultivés, viandes boucanées...) et des matières premières d'usages courants (sel, matériaux lithiques...), connaissent un développement important avec la période Formative, qui voit un accroissement démographique des populations, l'occupation sédentaire de nouveaux territoires, et la probable apparition de voies de communication pérennes (Burger 1995). Comme en témoigne la présence, dans l'ensemble des niveaux d'occupation du site de Cerro Ñañañique, de vestiges de coquillages et de vertèbres de poissons, ainsi que de fragments de poteries de style côtier Paita, ces échanges entre populations distantes d'une centaine de kilomètres peuvent prendre, dans le courant du $\mathrm{I}^{\mathrm{er}}$ millénaire $\mathrm{BC}$, un caractère régulier et sans doute formalisé.

Il existe également des données fiables attestant, à cette époque, l'échange à longue distance d'un certain nombre de marchandises pouvant être classés globalement sous la rubrique de produits rares, exotiques ou somptuaires. Ces trafics ont vraisemblablement concerné tant des vestiges animaux (coquillages, plumes, et vraisemblablement peaux), et minéraux (obsidienne, colorants, anthracite) que des produits manufacturés (éléments de parure, textiles, céramiques). D'autres produits végétaux, non conservés, peuvent probablement être inclus dans cette liste, en particulier les 
plantes psychotropes et hallucinogènes (coca, cactus San Pedro, liane Ayahuasca), ainsi que des matières d'usage culi-naire, médical ou technique (iodine, condiments, teintures, colorants...).

Ces échanges à longue distance, attestés dès la seconde moitié du $\mathrm{III}^{\mathrm{e}}$ millénaire $\mathrm{BC}$, s'accentuent vraisemblablement dans le courant du millénaire suivant, pour donner lieu à des systèmes établis, suivant des voies de communication relativement fixes, à partir de 1000 BC. Dans le sens nord-sud, ils peuvent avoir emprunté trois voies principales : la route maritime, par cabotage ; une voie terrestre, traversant un long désert côtier, entrecoupé d'oasis correspondant aux vallées irriguées ; et une voie des crêtes, longeant la cordillère orientale, suivant un tracé proche de ce qui sera le chemin inca. Dans le sens ouest-est, chacune des vallées côtières permettait d'accéder à une zone andine proche, mais le franchissement des Andes et l'accès au versant oriental amazonien est, dans plusieurs régions, difficile sinon impossible. Notre zone d'étude, caractérisée par la moindre amplitude des élévations de la chaîne andine, constitue, de ce point de vue, un des secteurs privilégiés pour les échanges avec les tributaires de l'Amazone. Une certaine spécialisation de ces voies et sites d'échange est probable. Ainsi les coquilles de Spondylus, provenant des eaux chaudes équatoriales, qui faisaient l'objet d'un trafic à longue distance jusque dans les Andes centrales (Chavín de Huantar) (Burger 1995, Lumbreras 1993), sont relativement abondantes sur les sites des andes du sud équatorien, tels Cerro Narrio (Collier, Murra 1943) et Catamayo (Guffroy et al. 1987), alors que seuls quelques rares fragments, de petites dimensions, ont été collectés à Cerro Nañañique.

\section{L' architecture}

La présence de constructions monumentales d'usages cérémoniels et civiques est très généralement considérée comme la donnée témoignant avec le plus d'évidence de la distance culturelle existant entre les populations des régions équatoriales et celles des Andes et de la côte, nord et centrale, du Pérou. La caractérisation de la présence sur le site de Cerro Ñañañique de plates-formes et de structures monumentales, comparables à celles des vallées plus méridionales (Fig. 7), a déjà fait reculer cette supposée frontière de plus de $300 \mathrm{~km}$ vers le nord.

Or, les conditions de découverte de l'occupation formative de Cerro Nañañique illustre parfaitement le caractère aléatoire des recherches, et les difficultés d'interprétation qui en découlent. Avant notre passage en juillet 1986, en compagnie de l'équipe d'archéologues de la PUC, le site, entouré par une ville de plusieurs dizaines de milliers d'habitants, avait fait l'objet d'une prospection, en 1979, et de visites répétées d'archéologues depuis 1962. L'identification de la présence d'une occupation formative avait été considérablement facilitée, entre-temps, par les pluies diluviennes intervenues en 1983, à l'occasion d'un phénomène «El Niño », qui avaient fortement entaillé les sédiments de fondation des grandes plates-formes, mettant au jour d'importantes quantités de matériel céramique, enfoui depuis 3000 ans. Par ailleurs, si un incendie, qui précéda sans doute l'abandon du site, n'était pas intervenu au $\mathrm{V}^{\mathrm{e}}$ siècle $\mathrm{BC}$, nous n'aurions aucune connaissance de la nature des bâtiments en élévation construits sur certaines de ces plates-formes. L'architecture complexe en quincha (torchis) qui a permis d'élever des murs de cinq mètres de hauteur (pour une épaisseur 
de 0,30/0,40 cm), ainsi que la présence de revêtements de paroi, de banquettes et de colonnes en argile, seraient restées totalement inconnues. Faute d'une préservation accidentelle d'une partie des matériaux, sous l'effet du feu, des aménagements de même nature, ou même encore plus importants, ont sans doute été totalement détruits par ailleurs.

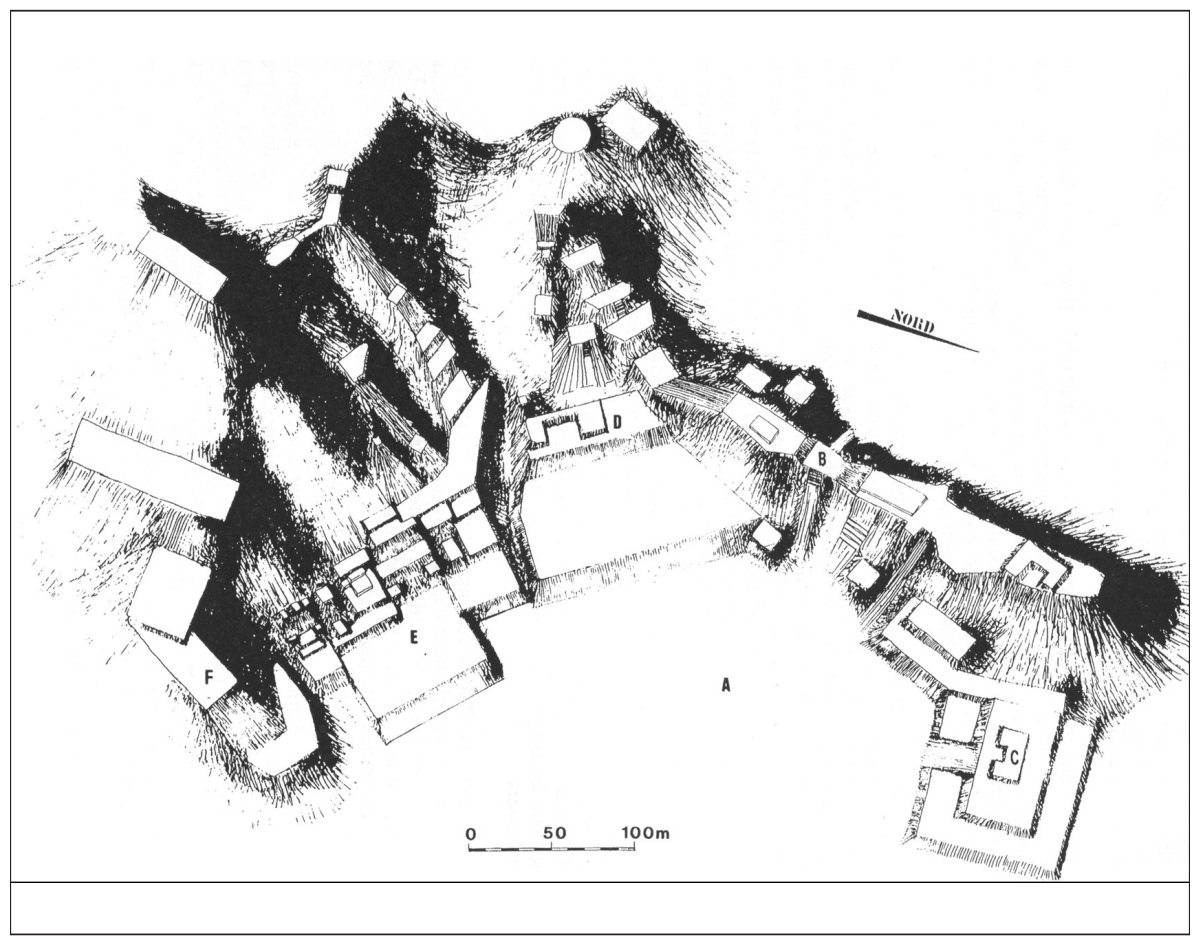

Fig. 7

Cerro Nañañique : Reconstitution axonométrique des principaux aménagements (hors bâtiments) au $\mathrm{VI}^{e}$ siècle $B C$.

Ces données illustrent clairement trois des principaux problèmes de l'analyse comparative des architectures anciennes : la nature différenciée des matériaux; les conditions, tout aussi diverses, de leur conservation ; le caractère aléatoire des découvertes archéologiques.

Dans notre zone d'étude, soumise depuis des millénaires à des conditions climatiques difficiles, les éventuelles constructions monumentales érigées en matériaux périssables (terre, briques crues, torchis) ont, dans des proportions élevées, disparu ; ou ont été si fortement érodées que leur caractérisation est devenue le plus souvent impossible. Ces difficultés, renforcées par la totale méconnaissance archéologique de vastes portions de territoire, rendent douteuse toute tentative de comparaison avec les régions de construction en pierre, ou de climat sec. Plusieurs découvertes, malheureusement trop souvent isolées, témoignent de l'érection de plates-formes et de possibles constructions monumentales dans le sud équatorien, depuis la période Formative ancienne (Real Alto) jusqu'au Formatif final (Sangay). 


\section{Autres sphères d'activités}

L'historique de la diffusion de la domestication et de l'élevage des camélidés, dans les Andes septentrionales, apporte également des éclairages intéressants sur le développement de ces sociétés formatives. Durant une première étape $\left(\mathrm{XIII}^{\mathrm{e}}\right.$-IX ${ }^{\mathrm{e}}$ siècles $\mathrm{BC}$ ), il existe une nette opposition entre les Andes centrales péruviennes, où les vestiges osseux de camélidés sont prédominants, et les Andes du sud équatorien, où ils sont totalement absents. Dans la zone intermédiaire (Pacopampa, Cerro Ñañañique...), la prédominance des cervidés dans l'alimentation carnée, n'exclut pas la présence d'un petit nombre d'ossements de camélidés. Cette présence minoritaire pourrait traduire une phase d'introduction de l'élevage, ou-plus vraisemblablement dans le cas de Cerro Ñañañique - la venue plus ou moins sporadique d'animaux, appartenant à des caravanes provenant des régions méridionales. Une évolution notable marque la fin de la période formative $\left(\mathrm{IV}^{\mathrm{e}}-\mathrm{III}^{\mathrm{e}}\right.$ siècle $\left.\mathrm{BC}\right)$, et le début de la période suivante $\left(\mathrm{II}^{\mathrm{e}}-\mathrm{I}^{\mathrm{er}}\right.$ siècle $\mathrm{BC}$ ), époque durant laquelle l'élevage de camélidés devient important dans les Andes du nord du Pérou et jusqu'au sud de l'Equateur (Bruhns et al. 1990).

La diffusion de certains objets, qui témoignent de pratiques et coutumes particulières, attestent également de relations qui ne respectent pas le supposé clivage nord-sud. C'est le cas des sceaux en céramique, probablement destinés à la décoration corporelle, présents sur la côte équatorienne, dans le Haut Piura et dans l'aire culturelle Cupisnique, mais apparemment absents dans toutes les régions andines d'altitude. Un autre objet, de fonction indéterminée (spatule, cuillère?), fréquemment fabriqué à partir d'un grand tesson retravaillé, est un vestige fréquent à Loja, dans le Haut-Piura et dans la région de Cajamarca.

\section{Les étapes suivantes}

Il nous est évidemment impossible de présenter ici l'analyse détaillée du développement de cette région « frontalière », postérieurement à la période Formative. Nos connaissances des différentes phases de l'histoire préhispanique restent elles-mêmes très confuses, particulièrement en ce qui concerne les périodes charnières qui voient une accélération des processus historiques. La majorité des données en notre possession tendent cependant à confirmer le caractère relativement singulier des occupations préhispaniques locales.

L'abandon du site de Cerro Nañañique, probablement lié à un incendie volontaire des principaux bâtiments (Guffroy et al. 1994 : 156-162), est associé à des changements importants qui se traduisent, entre autres, par l'apparition de nouvelles formes et décorations céramiques, extrêmement simplifiées par rapport à l'époque précédente. Une structure cérémonielle, de plus petites dimensions, semble avoir été édifiée, à cette époque, à proximité de Cerro Ñañañique. Cette phase, datée des $\mathrm{IV}^{-I I I}{ }^{\mathrm{e}}$ siècles $\mathrm{BC}$, correspond très probablement au moment de diffusion et d'extension maximale de l'idéologie Chavín (Burger 1995). Comme nous l'avons déjà signalé, ces influences atteignent, sous des formes diverses, les Andes équatoriennes (phase Catamayo D, Chaullabamba, Pirincay), et, probablement, les régions côtières voisines, durant la phase finale de la culture Chorrera (transition Chorrera / Bahia, Tabuchines...). 
Une importante rupture culturelle, dont les causes - naturelles et sociales - restent à déterminer, intervient, probablement, durant le $\mathrm{III}^{\mathrm{e}}$ siècle $\mathrm{BC}$. L'époque paraît également marquée par des mouvements de population, et une recrudescence des conflits, dont témoigne l'installation de nombreux sites fortifiés. Ces mouvements de migration semblent orientés depuis les zones périphériques (Andes septentrionales, versant amazonien ...) jusqu'aux régions précédemment prédominantes (côte nord Andes centrales). Bien que certains des nouveaux styles céramiques empruntent aux traditions antérieures, la rupture entre les deux époques est évidente. Elle est marquée, entre autres points, par le caractère plus fruste du matériel, et l'apparition de nouvelles formes de récipients. L'effondrement des systèmes socio-politiques formatifs pourrait avoir facilité l'intrusion de groupes de population à croissance démographique plus importante, attirés par les zones de fort développement. Dans le Haut Piura, cette phase (Chapica) est caractérisée par la présence de deux ensembles de matériel témoignant de relations avec les groupes installés à l'époque dans les Andes lojanaises, et dans tout le secteur oriental (Huacabamba, Chinchipe, Bagua), mais aussi avec les traditions contemporaines de la côte nord du Pérou (Huanchaco).

Les données, plus abondantes, concernant la phase suivante, Vicús ( ${ }^{\mathrm{I} r} \mathrm{BC}$ $\mathrm{VI}^{\mathrm{e}} \mathrm{AD}$ ) (Kaulicke 1991), confirment le caractère singulier des occupations humaines de ce secteur du Haut Piura, et prolongent les situations observées pour la pàriode Formative. Alors que le matériel céramique utilitaire de ce secteur est semblable à celui utilisé sur une assez vaste aire géographique, comprenant la région côtière (phase Sechura), et probablement les secteurs andins voisins ; il existe un autre style contemporain (Vicús) de grande qualité artistique, dont la distribution est limitée à un secteur réduit du Haut-Piura. La grande majorité de ce matériel a été mis au jour à l'occasion du pillage de sépultures, qui contenaient également d'importantes quantités de pièces de métallurgie et d'orfèvrerie. Dans ce même secteur, d'abondants vestiges attestent de la présence, continue et simultanée, de groupes porteurs de traditions culturelles méridionales (Cajamarca, Mochica) (ibid.), dont aucune trace n'a été retrouvée plus au nord. Comme c'était déjà le cas durant la période Formative, le caractère singulier de l'occupation de ce secteur paraît étroitement lié à sa position géographique, et à son implication dans des circuits économiques d'échanges à longue distance.

Les sociétés installées dans les Andes plus septentrionales semblent avoir évolué localement, et avoir été moins affectées par les phénomènes de crise et les ruptures culturelles. Comme nous l'avons précédemment indiqué, cette période voit l'introduction, dans cette zone, de l'élevage des camélidés et de la production métallurgique. Ces activités ont sans doute contribué à une croissance démographique, qui se traduit par l'occupation de nouveaux territoires (Guffroy et al. 1987), et, peut être, des mouvements de population vers le sud et l'est. Dans le bassin du rio Catamayo, les traditions formatives antérieures ont généré de petits ensembles céramiques distincts, attestant de sociétés, sans doute, engagées dans des relations d'échange, mais, avant tout, centrées sur l'exploitation de petits territoires aux caractères écologiques relativement homogènes.

D'importants changements, probablement précédés d'une période de conflits aboutissant à un remplacement de population, interviennent dans la province de Loja, postérieurement au VII ${ }^{\mathrm{e}}$ siècle de notre ère. Les nouveaux arrivants, qui ont occupé la région jusqu'à la conquête espagnole, où ils apparaissent dans les textes des chroniqueurs sous le nom de Paltas, paraissent s'être rattachés à la famille linguisti- 
que Jivaro, également installée sur le versant amazonien proche (Bracamoros). Ces groupes semblent avoir partagé une forme d'organisation sociale, tribale, un mode de vie, et des croyances, sensiblement différents de celles des autres populations andines. Leur arrivée dans la province de Loja est susceptible d'avoir fait disparaître les systèmes de relations et d'échange anciens, qui s'étaient maintenus jusqu'alors, et d'avoir introduit, de manière durable, une rupture, qui sera à l'origine des frontières postérieures. Durant toute cette période, le Haut-Piura paraît évoluer en périphérie de la zone d'influence de l'empire Chimu, qui a remplacé les structures politiques mochicas.

Les conquêtes inca (aux alentours de 1470), puis espagnole (1532), ont profondément perturbé, jusqu'à faire disparaître, les traditions culturelles précolombiennes locales. L'éloignement des pouvoirs centraux a alors fortement contribué au sousdéveloppement économique et social de ces territoires. De nombreuses données historiques attestent, cependant, que cette région a connu, à l'époque moderne, une série de développements économiques et sociaux singuliers, liés à sa position intermédiaire et à l'exploitation des ressources d'origines et de natures variées : gisements aurifères, aux $\mathrm{XVI}^{\mathrm{e}}$ et XVII ${ }^{\mathrm{e}}$ siècles, exportation de la cascarilla (quinquina), au XVIII ${ }^{\mathrm{e}}$, trafic de stupéfiants (cocaïne), au $\mathrm{XX}^{\mathrm{e}}$.

\section{CONCLUSIONS PROVISOIRES}

Il ressort de ces données que l'historique des occupations précolombiennes de cette région de basses Andes semble contredire, en majeure partie, sa caractérisation comme une barrière naturelle, difficilement franchissable, affectée d'un sousdéveloppement, économique et culturel, chronique. Sa position intermédiaire, propice aux échanges, paraît avoir joué un rôle important à plusieurs moments de la préhistoire, dont la période Formative. L'isolement socio-culturel d'une partie de cette région semble être apparu relativement tardivement, (postérieurement au VIII ${ }^{\mathrm{e}}$ siècle $\mathrm{AD}$ ), sous l'effet de l'arrivée de nouvelles populations, de probable origine amazonienne. Il a été clairement renforcé par les divisions administratives postérieures.

Parler de frontière en l'absence de données linguistiques ou sociopolitiques fiables constitue un exercice à haut risque. Or, dans notre cas d'étude, et en ce qui concerne plus généralement les situations " préhistoriques », le premier registre est définitivement perdu, alors que le second ne peut-être restitué qu'au moyen d'analyses complexes, pour lesquelles les vestiges sont encore le plus souvent trop rares ou trop peu explicites. Prôner dans ces conditions l'existence de frontières anthropo-géographiques, aux caractères et effets plus ou moins figés, constitue, à notre avis, une facilité de réflexion, qui reproduit trop souvent les idées préconçues, héritées des géographes des siècles derniers et des conditions de développement modernes. Attribuer un caractère systématiquement défavorable à certaines contraintes écologiques ne permet pas la prise en compte des évolutions susceptibles d'être intervenues sur le temps long, ni du caractère singulier des adaptations humaines. L'analyse des potentialités des environnements, et de leurs capacités en terme de ressources, ne peut constituer un exercice objectif intemporel, et doit être effectuée au regard des états de développement et des organisations sociales de chaque époque. 
L'étude conceptuelle des modes de fonctionnement de ces régions intermédiaires, ou périphériques, reste également, pour l'essentiel, à réaliser. Les données présentées dans ce texte témoignent, en tout cas, d'une complexité de situations qui s'accommode mal de la vision simpliste d'une zone tampon, déterminée par des conditions écologiques. Comme nous l'indiquions déjà en 1987 (ibid. : 342) : la question de l'influence des caractéristiques environnementales sur le peuplement et son évolution semble admettre des réponses diverses. Les contraintes géographiques sont peu opérantes dans les périodes où s'exercent des phénomènes sociaux qui transcendent l'espace et ses contingences, mais elles peuvent être déterminantes dans les périodes de repli, lorsque les forces sociales les utilisent à leur profit, et qu'elles justifient la singularité culturelle. Entre ces deux tendances extrêmes, clairement présentes dans les Andes, le rapport au milieu naturel peut revêtir des formes variées. Les limites frontalières sont l'affaire quotidienne des hommes, de leurs pouvoirs et de leurs idéaux, fruits d'interactions où l'élément écologique joue un rôle important mais pas toujours essentiel.

Il convient également de noter que plusieurs des situations et modalités de développement précédemment décrites, semblent être comparables, sous des formes bien évidemment spécifiques, à des développements historiques recensés dans d'autres régions du monde. Ainsi, plusieurs des caractères susceptibles d'expliquer l'implantation et la croissance du centre civico-cérémoniel de Cerro Nañañique rappellent la situation observée, en Europe, à partir du $\mathrm{XV}^{\mathrm{e}}$ siècle $\mathrm{BC}$, à la fin de l'âge du Bronze. Le développement, à cette époque, des principautés celtiques paraît en effet résulter de leur position intermédiaire entre le monde méditerranéen et l'aire baltique, source de matières premières recherchées. On observe également, dans cette région du globe, des évolutions postérieures diverses, témoignant de la relative fragilité de ces systèmes, dépendants d'éventuels changements de voies de communication, mais aussi des évolutions sociales affectant l'un ou l'autre des termes de l'échange. Des situations semblables ont existé dans d'autres régions du monde, telles les zones sahéliennes d'Afrique et du Moyen-Orient. Plus globalement, le processus historique, qui marque l'apparition et la chute de la première grande civilisation andine « Chavín », rappelle également des situations intervenues dans diverses régions et à diverses époques de l'ère pré-industrielle.

\section{RÉFÉRENCES BIBLIOGRAPHIQUES}

ALVA W.

1986 Cerámica temprana en el valle de Jequetepeque, norte del Perú, Materialen zur Allgemeinen und Vergleichenden Archäologie 32, Bonn : KAVA : 196 p.

BENNET W.C.

1948 The Peruvian co-tradition, in BENNET W.C. (éd.), A reappraisal of Peruvian Archaeology, Menasha, Wis. : Published jointly by the Society for American Archaeology and the Institute of Andean Research : 1-7. 
BRUHNS K., BURTON J., MILLER G.

1990 Excavations at Pirincay in the Paute valley of southern Ecuador, Antiquity 64/n $243: 221-233$.

\section{BURGER R.}

1984 Archaeological areas and prehistoric frontiers : the case of Formative Peru and Ecuador, in BROWMAN D., BURGER R., RIVERA M.A. (éds), Social and Economic Organization in the prehispanic Andes, Oxford : BAR International Series 194 : 33-64.

1995 Chavin and the origins of Andean civilizations, London : Thames and Hudson : 248 p.

COLLIER D., MURRA J.

1943 Survey ans excavations in southern Ecuador, Field Museum of Natural History, anthropological series 35, Chicago : $105 \mathrm{p}$.

DEBOER W.

1995 Ceramic asemblage variability in the Formative of Ecuador and Peru, Paper presented to the session «Archaeology of Formative Ecuador », Dumbarton Oaks conference, October 7-8 1995, Washington DC : Dumbarton Oaks : 23 p.

GOMIS D.

1999 La cerámica formativa tardia en la sierra austral del Ecuador (Cañar, Azuay y Loja) : unidad territorial y particulares regionales, in LEDERGERBER J.A. (éd.), Formativo suramericano, Quito : ABYA-AYALA : 139-159.

GUFFROY J., ALMEIDA N., LECOQ P., CAILLAVET C., EMPERAIRE L., ARNAUD B.

1987 Loja préhispanique. Recherches archéologiques dans les Andes méridionales de l'Equateur, Paris : Ed. ADPF : 342 p.

GUFFROY J., en collaboration avec BARAYBAR J., CARDOZA C.R., CARLIER G., CLEMENT G., DONZE P., EMPERAIRE L., FOURNIER M., GIRARD M., MARCH R., ORTLIEB L., SOSA G., WATTEZ J.

1994 Cerro Nañañique : un établissement monumental de la période formative, en limite de désert (Haut Piura, Pérou). Paris : ORSTOM : 440 p.

\section{GUFFROY J.}

1995 L'influence des conditions environnementales sur le peuplement et le développement dans un secteur intermédiaire des Andes. Critique d'un certain déterminisme écologique, in MARLIAC A. (éd.), Milieux, sociétés et archéologues, Paris : Ed. KARTHAL/ORSTOM : 79-118.

\section{HOCQUENGHEM A.M.}

1991 Frontera entre áreas culturales nor y centroandinas en los valles y costa del extremo norte peruano, Bulletin de l'Institut Français d'Etudes Andines 20/2 : 309-348.

HOCQUENGHEM A.M., IDROVO J., KAULICKE P., GOMIS D.

1993 Bases del intercambio entre las sociedades norperuanas y surecuatorianas : una zona de transicion entre 1500 A.C. y 600 D.C., Bulletin de l'Institut Français d'Etudes Andines 22/2 : 443-466.

\section{KAULICKE P.}

1991 El periodo Intermedio Temprano en el Alto Piura : avances del proyecto arqueologico Alto Piura (1987-1990), Bulletin de l'Institut Français d'Etudes Andines 20/2 : 38-422.

LUMBRERAS L.G.

1993 Chavin de Huantar. Excavaciones en la galeria de las Ofrendas, Materialen zur Allgemeinen und Vergleichenden Archäologie 51, Mainz am Rhein : KAVA : 461 p.

\section{TELLENBACH M.}

1995 Las relaciones entre Ecuador y Peru en el Formativo, in GUINEA M., BOUCHARD J.F., MARCOS J. (éds), Cultura y medio ambiente en el area andina septentrional, Quito : ABYA-AYALA : 279321 . 